Research Paper

\title{
High Fat High Calories Diet (HFD) Increase Gut Susceptibility to Carcinogens by Altering the Gut Microbial Community
}

\author{
Heiying Jin ${ }^{\bowtie}$, Chunxia Zhang \\ Department of colorectal surgery, The Second Affiliated Hospital of Nanjing University of Chinese Medicine, 23Nanhu Road, Nanjing 210017, China \\ $\triangle$ Corresponding author: Heiying Jin. E-mail: jinheiying@hotmail.com; Tel: 86-25-83291202 \\ (c) The author(s). This is an open access article distributed under the terms of the Creative Commons Attribution License (https://creativecommons.org/licenses/by/4.0/). \\ See http://ivyspring.com/terms for full terms and conditions.
}

Received: 2020.01.01; Accepted: 2020.02.14; Published: 2020.04.07

\begin{abstract}
Objective: To investigate the risk of colorectal cancer and its relationship with colonic flora and microenvironment under high-fat and high-calorie diet. Methods: Wistar rats were used to study, and they were given normal diet, high-fat diet, and dimethyl hydrazine (DMH) to induce the occurrence of colorectal cancer. Then observe the difference in tumor formation and the relationship among microbial community, inflammatory factors and metabolism. Results: No tumors were found in the normal diet group (G1) and the high-fat diet group (G3). Four nodules were found in the four rats in the normal diet + DMH group (G2) and 8 cancerous nodules were formed in 7 rats (70\%) from high-fat diet + DMH group (G4). Cholesterol and TNF- $\alpha$ increased, IL-1, IL-6 and LEP decreased in the high-fat diet group. The difference was statistically significant. In the cancer-inducing group, only the difference in cholesterol was statistically significant. Compared with the normal diet group (G1) and the high-fat diet group (G3), the rat's gut bacterial abundance was not significantly different, but the gut flora structure was significantly changed. The content of Candida in the intestinal tract of rats in the high-fat diet group was reduced $(P=$ $0.015)$, while the content of Verrucomicrobia increased $(P=0.035)$; In the comparison of genus content, Ruminococcus, Candida, Saccharibacteria genera incertae sedis, Enterobacter, Clostridium IV, Enterococcus, Enterorhabdus, Acetivibrio, Adlercreutzia, Lactococcus, etc., decreased significantly, while Akkermansia, Warthococcus, Staphylococcus, Butyricimonas, Clostridium XVIII, etc. increased significantly.

Conclusion: This study found that high-fat, high-calorie diet can increase the susceptibility of the intestine to carcinogenic factors. The reason may be that the high-fat diet causes the body to appear inflammatory states and microbial community imbalance, especially rumenococcus, candida, Saccharomyces, Enterobacter, Clostridium IV, Enterococcus, Enterobacter, Vibrioaceticus and other genus reduction are important links. Exploring ways to improve these floras is an important factor to improve the resistance of the intestinal tract to cancer-inducing agents.
\end{abstract}

Key words: microbial community; colorectal cancer; susceptibility; prevention

\section{Introduction}

High-fat and high-calorie diet is an important factor in the development of colorectal cancer, but the exact mechanism is still controversial. It is generally believed that high-fat and high-calorie diet causes hyperlipidemia and obesity, puts the body in a chronic inflammatory state, and may promote the intestine and thus stimulate the occurrence of tumors [1-2]. Recent studies have shown that high-fat and high-calorie diets can cause changes in the abundance and types of microbial community, which may be an important cause of colorectal cancer [3-4]. In our previous study, $80 \%$ of the 159 colorectal patients have hyperlipidemia[4].We also used tea extract to treat patients with hyperlipidemia, the serum lipid level ,insulin resistance and the inflammation factor such as TNF- $\alpha$ and IL-1 and found out that decreases[5-6]. 
However, we still did not know what is the exact the pathway of the high-fat and high-calorie diet, body inflammation state, changes in the intestinal flora and colorectal cancer. Is the colorectal cancer caused by changes in the intestinal flora itself? Or is it due to changes in the intestinal flora that the body's resistance to carcinogens changes, thereby making colorectal cancer more likely? There is no conclusion yet. This study investigated the relationship between changes in intestinal flora of rats under high-fat and high-calorie diet and tumorigenicity of carcinogens, and further explored the relationship between changes in intestinal flora under high-fat diet and carcinogenicity of carcinogens to provide new ideas for the prevention and treatment of colorectal cancer.

\section{Materials and methods}

\section{Feeding and grouping of experimental animals}

40 SPF Wistar rats, male and female, 5-6 weeks old, weight $200 \mathrm{~g}$ (animal certificate number: NO.11400700154060, experimental animal production license number: SCXK (Su) 2011-0003). Animal experiments were approved by the Animal Ethics Committee of the School of Medicine, Southeast University.

Animal diet was cobalt 60 radiation-sterilized pellets for rats and mice (Nanjing Jiangning Qinglongshan feed Company), high-fat feed (formulation: $20 \mathrm{~g}$ lard, $5 \mathrm{~g}$ cholesterol, $10 \mathrm{~g}$ Tween 80 , add water to $50 \mathrm{ml}$ ).High-fat diet group: daily high-fat feeds were administered to the stomach. Gavage daily with warm saline in normal diet group.

Experimental animal grouping:

G1 group: normal diet group (control)

Group G2: normal diet + DMH

G3 group: high-fat diet group (control)

Group G4: high-fat diet + DMH

\section{Animal treatment}

At the 4th week of feeding, the mice were injected subcutaneously with dimethyl hydrazine $(\mathrm{DMH})$ at $30 \mathrm{mg} / \mathrm{kg}$ twice a week. Dosing was continued for 8 weeks. The other groups were given weekly subcutaneous injections of the same amount of normal saline. Rats were weighed once a week to adjust the amount of DMH. At the 20th week of feeding, the rats were sacrificed.

\section{Detection of lipid metabolism and inflammatory factors}

The blood was removed by eyeballs, and the plasma was separated by centrifugation, and the blood glucose and blood lipid metabolism indicators (total cholesterol, triolein, HDL, LDL) were detected.
Inflammatory factors (IL-1, IL-6, COX-2) and cytokines (TNF- $a$, LEP) were detected by enzyme-linked immunosorbent assay.

\section{Fecal specimens and tissue specimens are retained}

Before putting the animals to death, take $0.1 \sim$ $0.3 \mathrm{~g}$ of fresh feces by massaging the abdomen, and immediately put them into liquid nitrogen and then store them in $-80{ }^{\circ} \mathrm{C}$ refrigerator.

The rats were dissected, and the large intestine was cut freely and fully. The ascending colon, transverse colon, descending colon, and rectal intestinal mucosa were separated and stored in a $-80^{\circ}$ $\mathrm{C}$ refrigerator. The number and size of colon tumor in the rats were calculated and measured. Tumors or suspected tumors were completely removed and divided equally 2 parts, one part is stored in 10\% formaldehyde and the other part is quickly frozen and transferred to $-80{ }^{\circ} \mathrm{C}$ refrigerator for storage. The intestine is cut open longitudinally, flattened between two layers of filter paper and fixed at $10 \%$ Neutral buffered formalin solution. Tumor size was measured and HE stained for pathological analysis.

\section{Research methods of coliform flora [8-9]}

Frozen fresh rat feces samples were quickly weighed and $0.1 \mathrm{~g}$ samples were taken and centrifuged to extract DNA samples after rewarming.1ul MD microplate reader (MD company in the United States) was used to quantitatively detect the quality of DNA samples. After extraction, the DNA samples were expanded by 16sPCR (primer: 16s) -341F: CCTACGGGNGGCWGCAG; 16s- 341F: CCTACGGGNGGCWGCAG), the samples were sequenced according to the Illumina Miseq high-throughput sequencer usage guide. After running for 3-5 days, the original data was converted to Fastq format, and the data quality reached more than Q30 80\%. In microbial diversity research, alpha diversity was measured by species richness (Richness, Chao, ACE) and diversity index (Shannon, Simpson); beta diversity analysis compares the similarity of species composition of samples, including cluster Class analysis and ranking analysis. The species abundance ratio chart counted each sample according to the boundaries, phylum, class, order, family, genus, and species level of the taxonomic unit of the species. Kruskal / wilcoxon rank sum test, similarity analysis (Anosim), Permanova substitution variance test, etc. were used to analyze the abundance and difference between species.

\section{Statistical methods}

SPSS18.0 statistical software was used to analyze the data. The measurement data were all Mean \pm SD. 
The comparison of multiple groups of data was performed by single factor analysis of variance. The comparison between groups was performed by LSD- $t$ test. The repeated measurement data was analyzed by repeated measurement analysis of variance. When $\mathrm{P}$ $<0.05$, the difference was statistically significant.

\section{Results}

\section{Tumor formation characteristics of rats in each group}

Rats gradually gained weight, but there was no significant difference between the 4 groups.There was no tumor formation in the normal diet group (G1) and high-fat diet group (G3). Four rats (40\%) in the normal diet $+\mathrm{DMH}$ group (G2) formed 4 nodules, and the tumor weight was $0.06 \pm 0.05 \mathrm{~g}$. Seven rats $(70 \%)$ from fatty diet $+\mathrm{DMH}$ group (G4) formed 8 cancerous nodules with a tumor weight of $0.05 \pm 0.03 \mathrm{~g}$. Compared with the normal diet cancer-inducing group, the high-fat diet cancer-inducing group had increased intestinal tumor formation (70\% VS 40\%) (Figure 1).

\section{Effects of high-fat diet on metabolism and inflammatory factors}

Animals in the high-fat diet group increased cholesterol and TNF- $\mathrm{a}$, while IL-1, IL-6, and LEP decreased. The difference was statistically significant. In the DMH-inducing group, only the difference in cholesterol was statistically significant (Table 1).

\section{Effect of high-fat diet on gut flora}

Compared with the normal diet group (G1) and the high-fat diet group (G3), the intestinal bacterial abundance of rats was not significantly different, but the intestinal flora structure was significantly different (ANOSIM $\mathrm{R}=0.2884, \mathrm{P}=0.011$; PERMANOVA $\mathrm{F}=3.750, \mathrm{P}=0.001)$.

Table 1: Comparison between lipid metabolism and serum inflammation corresponding groups $(n=40)$

\begin{tabular}{llllll}
\hline & G1 & G3 & P(G1vs G3) & G2 & G4 \\
\hline Glucose & $9.57 \pm 2.66$ & $6.89 \pm 0.64$ & 0.619 & $7.81 \pm 1.19$ & $7.40 \pm 1.72$ \\
Triglyceride & $0.93 \pm 0.30$ & $0.58 \pm 0.09$ & 0.366 & $0.58 \pm 0.26$ & 0.909 \\
cholesterol & $6.02 \pm 1.04$ & $7.38 \pm 1.61$ & 0.002 & $5.85 \pm 1.31$ & $0.70 \pm 0.23$ \\
LDL & $6.57 \pm 2.10$ & $5.32 \pm 3.24$ & 0.550 & $4.75 \pm 1.75$ & $0.19 \pm 2.65$ \\
HDL & $2.42 \pm 1.02$ & $2.07 \pm 1.14$ & 0.724 & $1.74 \pm 0.40$ & $6.70 \pm 2.86$ \\
IL-1 & $35.61 \pm 13.84$ & $24.83 \pm 12.89$ & 0.001 & $21.68 \pm 11.71$ & $2.68 \pm 1.45$ \\
IL-6 & $9.55 \pm 1.77$ & $7.52 \pm 1.57$ & 0.001 & $7.66 \pm 1.66$ & $21.49 \pm 10.84$ \\
LEP & $1.71 \pm 0.29$ & $1.36 \pm 0.55$ & 0.013 & $1.38 \pm 0.22$ & $7.03 \pm 1.25$ \\
TNF-a & $28.00 \pm 23.02$ & $45.73 \pm 13.23$ & 0.018 & $43.23 \pm 14.08$ & 0.105 \\
\hline
\end{tabular}

G1, normal diet group (blank control); G2, normal diet + DMH; G3, high fat diet group (blank control); G4, high fat diet + DMH

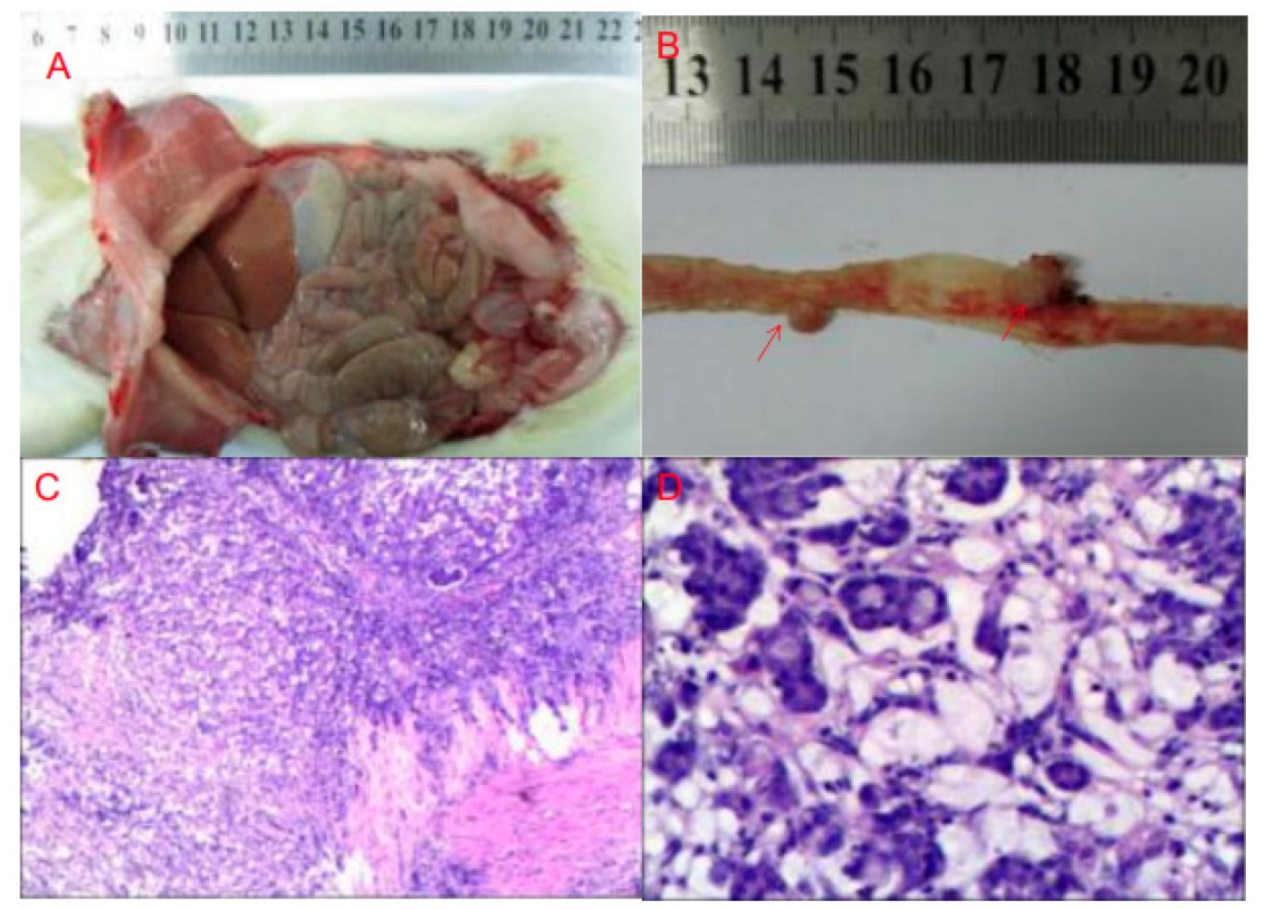

Figure 1. DMH-induced tumor formation in animal specimens and pathological HE staining results (A: gross finding B: cancer nodules red arrow showed C: cancer nodule $\times 200$ D: cancer nodule $\times 400$ ) 
In the comparison of the phylum, the content of Candidatus Saccharibacteria in the high-fat diet group (G3) was significantly lower than that in the normal diet group (G1) $(P=0.015)$, and the content of Verrucomicrobia was significantly higher than that in G1 group $(P=0.035)$ (Figure 2 ).

In the comparison of bacterial species (genus) content, the rats in the high-fat diet group (G3) had significantly less gut bacterial species than the normal diet group (G1): Ruminococcus, Candida, Saccharibacteria genera incertae sedis, Enterobacter, Clostridium IV, Enterococcus, Enterorhabdus, Acetivibrio, Adlercreutzia, Lactococcus, Streptococcaceae, Clostridiales_Incertae_Sedis_XI, Gallicola, Coriobacteriales, Vagococcus, Pseudoflavonifractor, Slackia, Erysipelotrichaceae incertae sedis, Gallicola, Helicobacter, Unclassified Aerococcaceae, Unclassified Bdellovibrionales, Unclassified Clostridiales, Unclassified Ruminococcaceae.

In the comparison of bacterial species (genus) content, the high-fat diet group (G3) rats had significantly increased gut bacterial species compared to the normal diet group (G1): Akkermansia, Verrucococcus, Staphylococcus, Butyricimonas, Clostridium XVIII, Leuconostocaceae, Weissella, Pseudomonas, Bifidobacterium, Anoxybacillus, Acinetobacter, Anoxybacillus, Escherichia/Shigella, Unclassified Brucellaceae. Compared with the G1 group, the content increased significantly (Figure 3).
Effect of carcinogen DMH on the gut flora of animals in normal diet group and high-fat diet group

Compared with G4 group, G2 group had no significant difference in gut bacterial abundance, but the intestinal flora structure changed significantly (ANOSIM $\mathrm{R}=0.292, \mathrm{P}=0.001$; PERMANOVA $\mathrm{F}=$ 3.697, $\mathrm{P}=0.001)$. In the comparison of bacteria categories, the contents of Actinobacteria and Candida in the G4 group were significantly lower than those in the G2 group (Figure 4).

In the comparison of bacterial species content, the bacteria that were significantly reduced in the G4 group compared to the G2 group were: Ruminococcus, Vibrio acetate, Adlercreutzia, Clostridium IV, Enterorhabdus, Erysipelotrichaceae incertae sedis, Facklamia, Leafworm, Gordonibacter, Helicobacter, Lachnospiracea incertae sedis, Lactococcus, Oligobacterium, Pseudoflavonifractor, Saccharibacteria genera incertae sedis, Saccharibacteria genera incertae sedis, Slackia, Nonclass, Unclassified Bacteroides, Unclassified Bdellovibrionales, Unclassified Clostridium, Unclassified Coriobacteriaceae and unclassified rumenaceae.

The bacteria that significantly increased in the G4 group compared to the G2 group were: Clostridium XI, Streptococcus pepticus, Ackermania, Micrococcus versicolor, Clostridium XVIII, Coprococcus, Streptococcus (Figure 5).

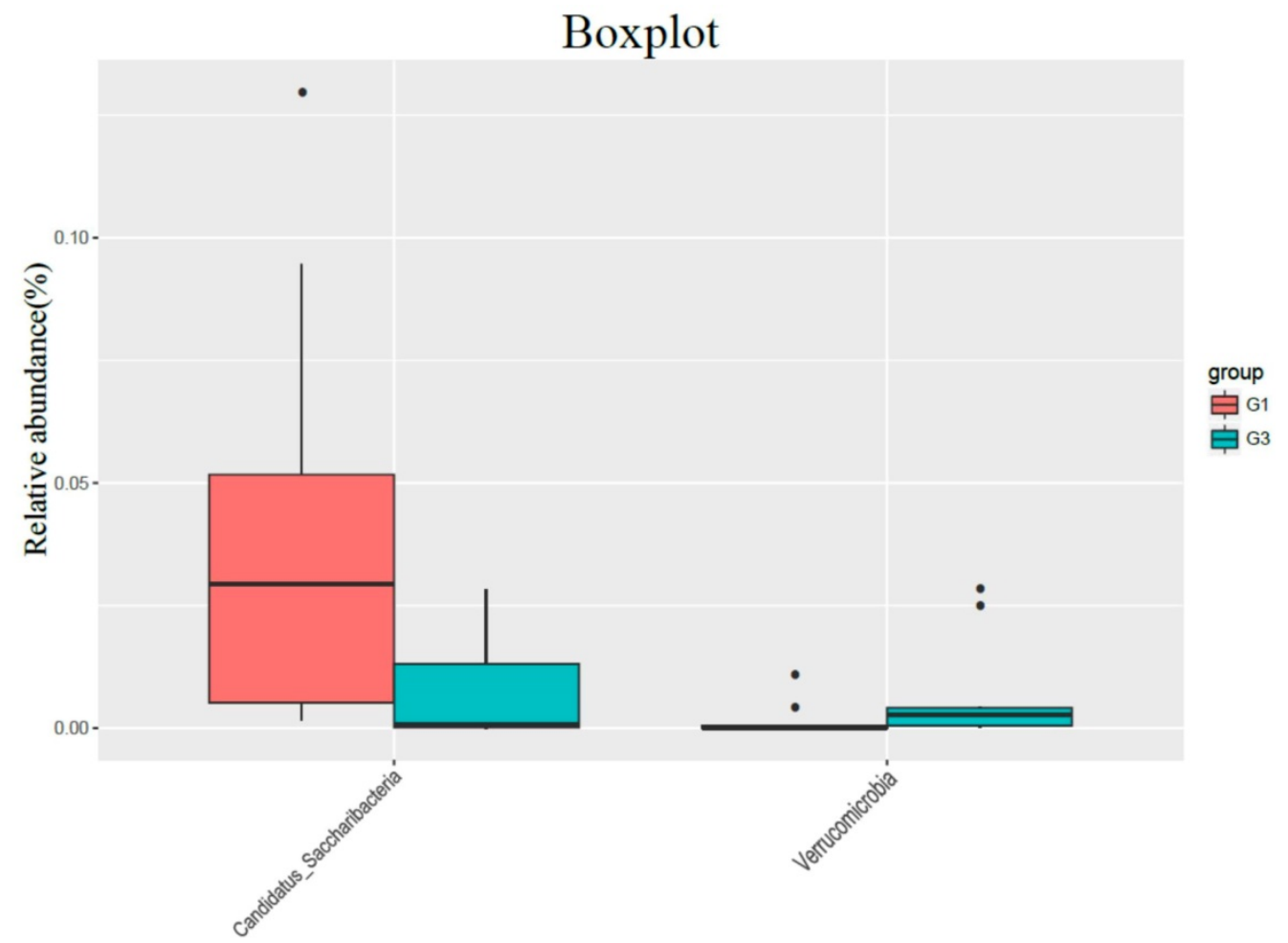

Figure 2. The influence of normal diet group (G1) and high-fat diet group (G3) on the flora 

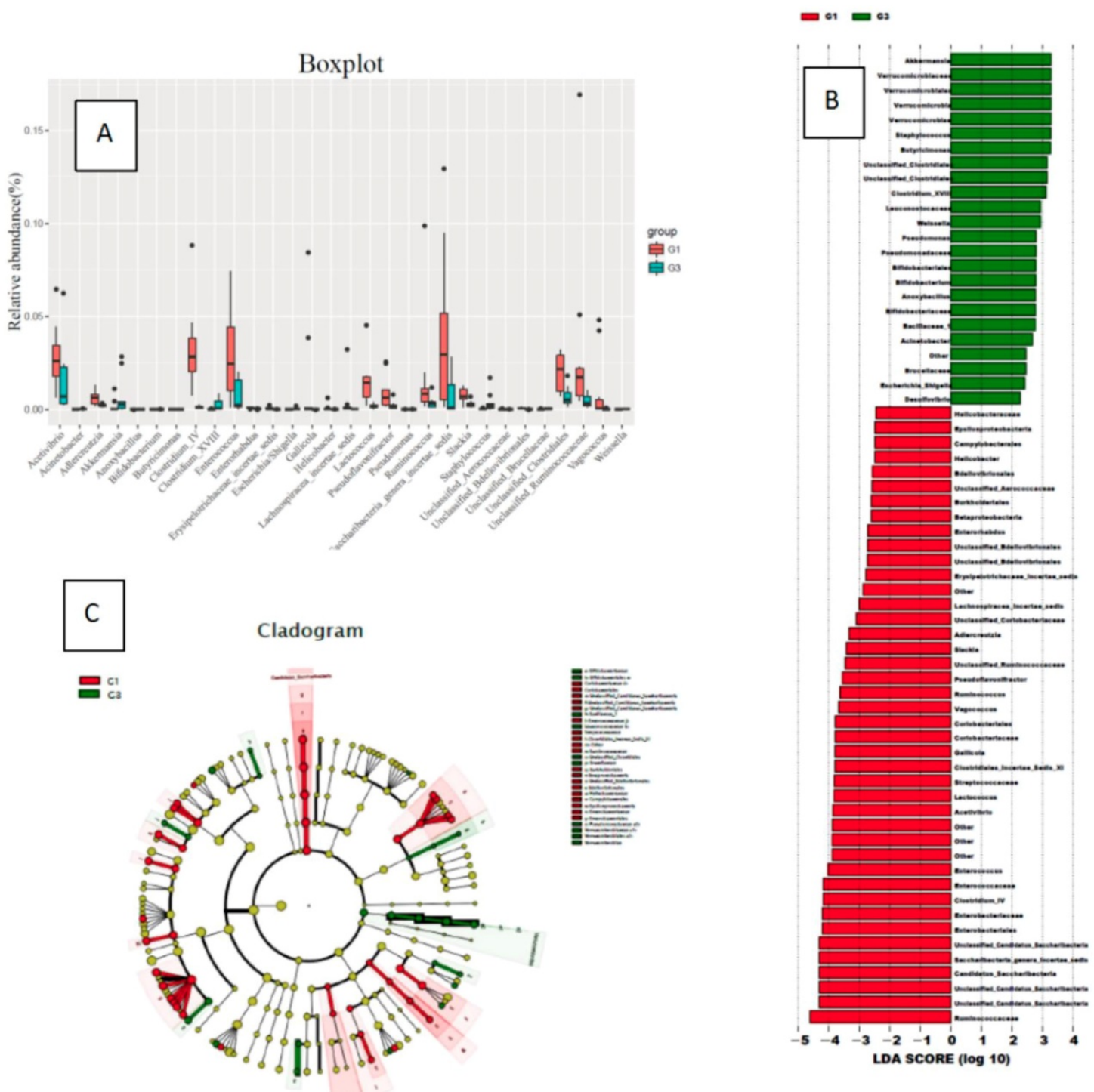

Figure 3. Changes in the species content of the bacterial flora in the normal diet group (G1) and the high-fat diet group (G3) (A: a comparison chart of the changes of each genus; B: an LDA chart of the changes of each genus; C: changes of each genus evolutionary branch diagram)

\section{Discussion}

High-fat and high-calorie diet plays an important role in the development of colorectal cancer, but there is no clear evidence on how high-fat and high-calorie diet can cause colorectal cancer [10-12]. High-fat and high-calorie diets cause hyperlipidemia in the body, which in turn causes the body to be in a chronic inflammatory state. But can high-fat and high-calorie diets alone cause the occurrence of colorectal tumors?

From the research in this group, compared with the normal diet group, the high-fat and high-calorie diet group had hyperlipidemia and inflammatory factors such as TNF-a increased.But after 20 weeks of observation, the animals did not develop colorectal cancer,which suggested that only high-fat, high-calorie diet will not cause colorectal tumor for a short period of time. In the literature, there have been few reports of the use of high-fat diet alone to induce colorectal tumors [13-14]. Current research suggests that a high-fat, high-calorie diet can cause the body to be in an inflammatory state, which may cause the body to be susceptible to cancer [15-17]. Tuominen et al. [18] studied AOM-induced colorectal cancer in animals using high-fat and high-calorie diets. If the high-fat and high-calorie diet was switched to a normal diet at the beginning of the cancer-inducing experiment, the risk of colorectal cancer in animals would not be significantly reduced. Therefore, the author believed that the high-fat and high-calorie diet played a role in colorectal tumor which may be an early stage affair. Xiu et al. [3] also considered that a high-fat, high-calorie diet can promote tumor growth in tumor-bearing animals. 


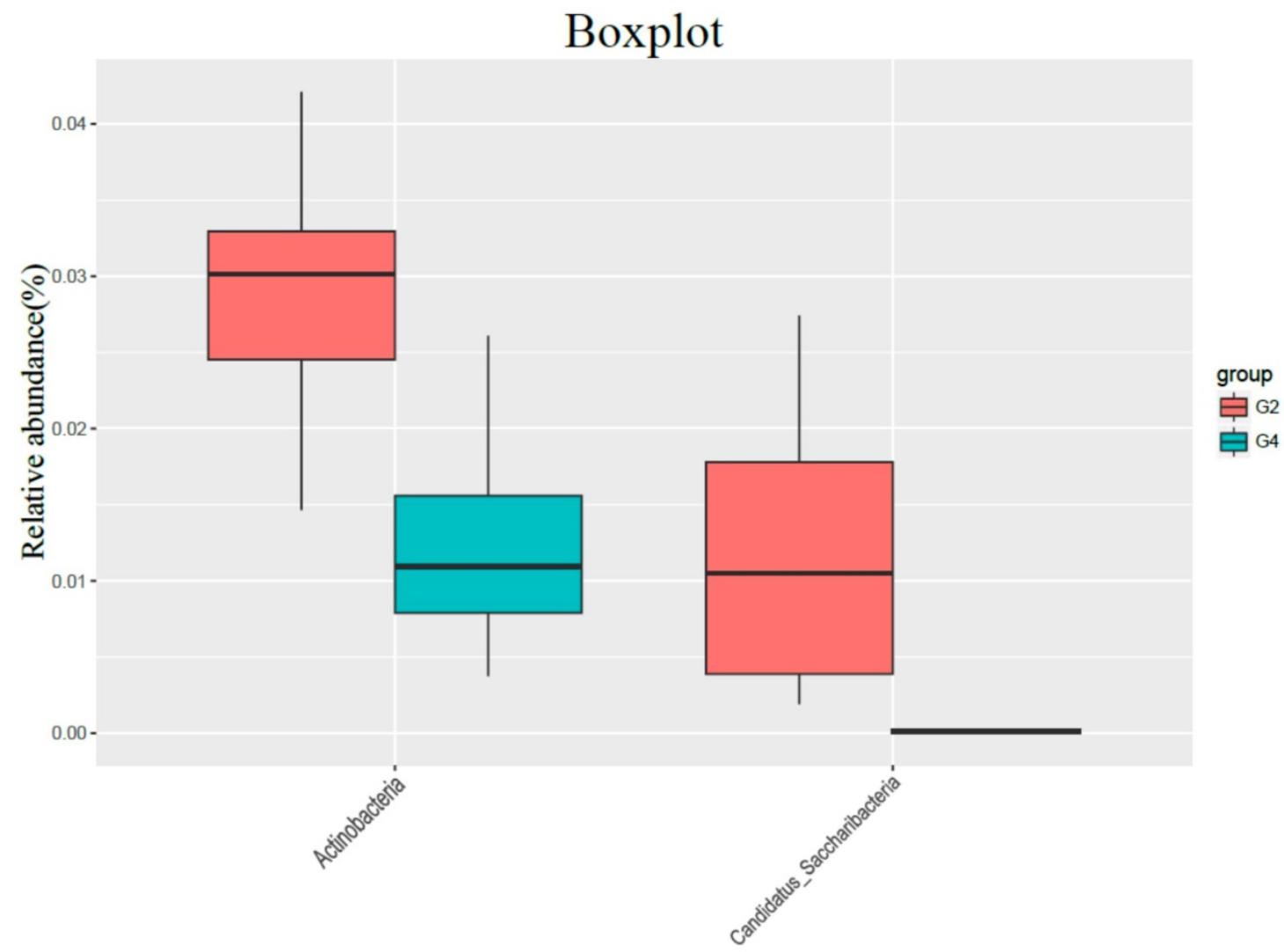

Figure 4. The influence of normal diet group (G2) and high-fat diet group (G4) on the flora

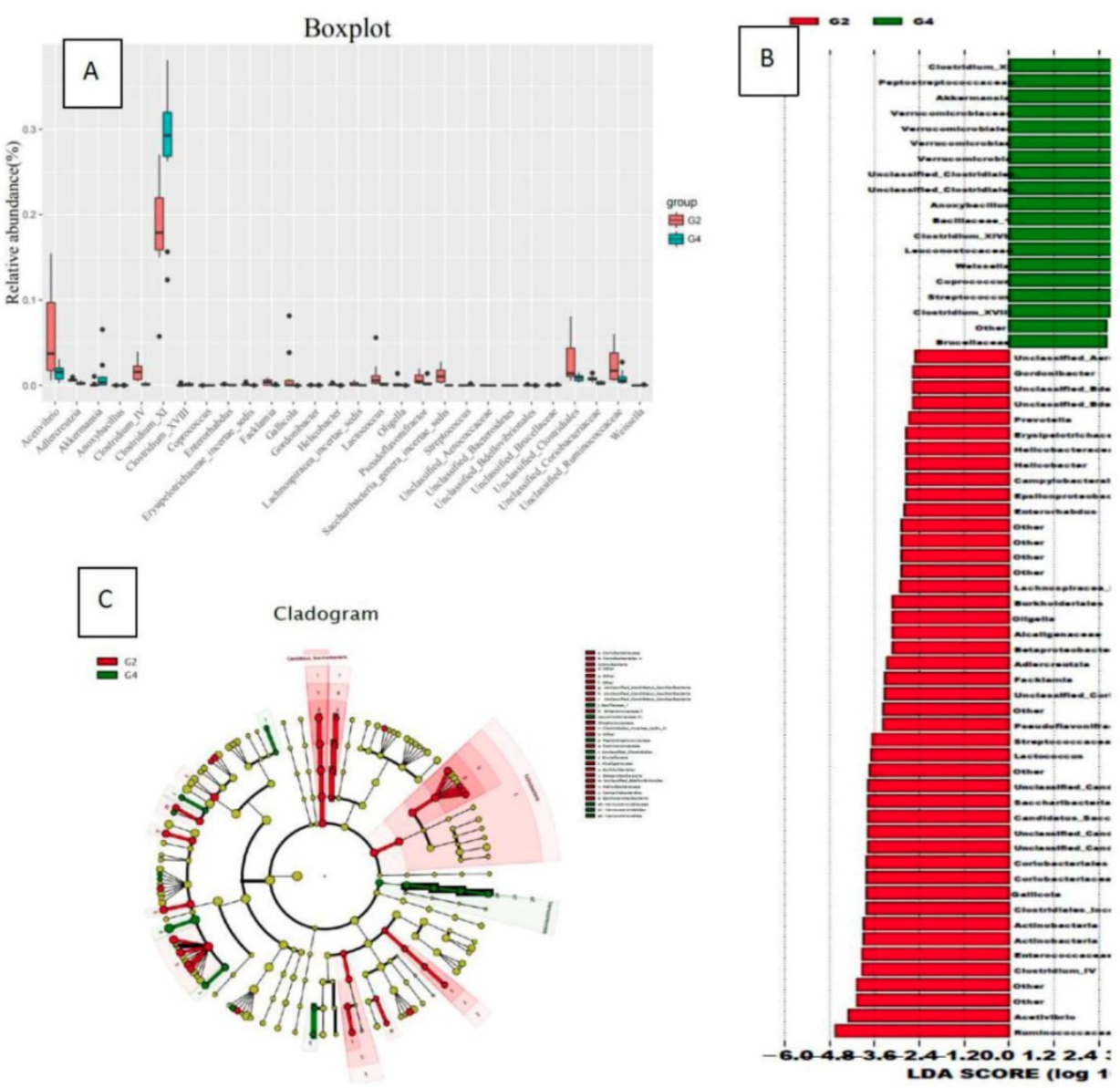

Figure 5. The effect of normal diet + DMH group (G2) and high-fat diet group + DMH group (G4) on the types of flora (Evolving branch diagram of changes in each genus) 
In general, hyperlipidemia caused by a high-fat, high-calorie diet may put the body in a chronic inflammatory state, increases the body's sensitivity to carcinogenic factors, and makes the body more prone to tumors [15-17]. In our study, the incidence of colorectal cancer in the normal diet group with $\mathrm{DMH}$ was $40 \%(4 / 10)$, while the incidence of colorectal cancer in the high-fat diet group with DMH was $70 \%$ $(7 / 10)$, and the tumor formation rate increased significantly. It implied that the high-fat and high-calorie diet causes the body to be in a chronic inflammatory state and may increase the sensitivity to carcinogens.

Modern research has shown that chronic inflammatory station leading to imbalance of the coliform flora may be an important cause of susceptibility to colorectal cancer [17-18]. In this group of studies, although the bacterial abundance of animals in the high-fat diet group did not change significantly, the types and distribution of bacteria changed significantly. In the comparison of gut microbiome, the content of candida in the intestine of rats in the high-fat diet group was significantly reduced compared with the normal diet group, while the content of Verrucomicrobia was significantly increased compared with the normal diet group. Verrucomicrobia is a kind of bacteria enriched in the intestinal mucosa, which will decrease in high-fat diets, obesity and other diseases. This study is similar to reports in the literature [19].

At the species level, rumenococcus, candida, saccharin, enterobacteria, clostridium IV, enterococcus, enterobacteria, vibrioaceticus, etc. were reduced in animals of the high-fat and high-calorie diet group. Most of these bacteria are already reported probiotics. The reduction of these bacteria may cause intestinal microenvironment disorders, and the intestinal mucosa barrier to resist external invasion will decrease, leading to increased susceptibility to carcinogenic factors [20-21]. After using $\mathrm{DMH}$, the proportion of rumenococcus, Candida, Clostridium IV, and Enterobacter bacteria in the high-fat diet group decreased further, further indicating that the decrease in the proportion of these bacteria is an important cause of the increased intestinal susceptibility to carcinogens the reason.

In the high-fat and high-calorie diet group, bacterial genera such as Akkermansia, Warthococcus, and Staphylococcus increased. The increase in the proportion of these bacterial genera may be related to the decrease in the proportion of other bacterial genera. In this group of results, the content of Akkermansia increased in high-fat and high-calorie diets [22-23], and the literature reported that in high-fat and high-calorie diets and metabolic diseases, proportion of Akkermansia may decrease. The results were different from those reported in the literature. The reason was not clear. It may be related to the significant decrease in the proportion of other bacteria and lead to increase Akkermansia relatively in this group of studies. Further research will be conducted to conform our outcome. After the use of DMH to induce cancer, bacteria such as Clostridium XI, Streptococcus digesta, Ackermania, Clostridium XVIII increased, suggesting that these bacteria play a harmful role in the intestinal, which may be the reason of the intestinal tract to carcinogens.

Due to the complexity of the intestinal flora, a series of bacteria rise and fall after a high-fat, high-calorie diet but how these bacteria work and what role they play is currently unclear. From the perspective of clinically regulating the intestinal microecology, selective fecal transplantation can be used to supplement the decreased bacteria, but there is currently no better way to remove the increased bacteria [24].Therefore, it is significant to explore the decreased beneficial bacteria and find ways to improve these.

In summary, this study shows that high-fat and high-calorie diets can increase the susceptibility of the intestines to carcinogenic factors, which may be due to high-fat diets leading to inflammatory states and intestinal flora imbalances, especially rumenococcus, candida, Saccharomyces, Enterobacter, Clostridium IV, Enterococcus, Enterobacter, Vibrioaceticus and other genus reduction are important links. Exploring ways to improve these flora is an important factor to improve the resistance of the intestinal tract to cancer-inducing agents.

\section{Abbreviations}

HFD: high-fat diet; DMH: dimethyl hydrazine; TNF: tumor necrosis factor; LEP: leptin; IL: interleukin; HDL: high density lipoprotein; LDL: low density lipoprotein; COX: cyclooxygenase.

\section{Acknowledgements}

The study is funded by Special foundation for key Research \& Development plans of Jiangsu Province (EB2019763), Jiangsu Nature Science Foundation (BK20191502).

\section{Competing Interests}

The authors have declared that no competing interest exists.

\section{References}

1. Daniel H, Gholami AM, Berry D, et al. ISME J. High-fat diet alters gut microbiota physiology in mice.2014;8:295-308.

2. Choi S, Snider AJ. Diet, lipids and colon cancer. Int Rev Cell Mol Biol. 2019;347:105-144. 
3. Xiu L, Yang Z, Zhao Y, et al. High-fatdiets promotecolonorthotopic transplantation tumor metastasis in BALB/c mice. Oncol Lett. 2019;17(2):1914-1920.

4. Si $\mathrm{ZH}$, Jin HY. Distribution characteristics and clinical value of lipid metabolism abnormality in colorectal cancer patients. World Journal of Chinese Digestion, 2011,19: 3542-3545

5. Zhu ZW, Jin HY, Wu Q. The effect of lotus leaf black tea on the syndrome of endogenous damp heat syndrome. Shandong Journal of traditional Chinese medicine, 2018;37: 989-991

6. Zhu ZW, Jin HY. Effect of lotus leaf black tea on lipid metabolism and colorectal cancer related molecules in patients with endogenous damp heat syndrome [J]. Shanxi traditional Chinese medicine, 2018;39: 467-469

7. Larsson SC, Wolk A. Meat consumption and risk of colorectal cancer: a meta-analysis of prospective studies. Int J Cancer, 2006;119(11):2657-2664.

8. Zhu Q, Jin Z, Wu W, et al. Analysis of the Intestinal Lumen Microbiota in an Animal Model of Colorectal Cancer. PloS ONE.2014;9(6): e90849

9. Dejea CM, Fathi P, Craig JM, et al. Patients with familial adenomatous polyposis harbor colonic biofilms containing tumorigenic bacteria. Science. 2018;359(6375):592-597.

10. Everard A, Geurts L, Caesar R, et al. Intestinal epithelial MyD88 is a sensor switching host metabolism towards obesity according to nutritional status. Nat Commun.2014;5:5648-5660.

11. Fritsche KL. The science of fatty acids and inflammation. Adv Nutr. 2015;6(3):293s-301s.

12. Liu Z, Brooks RS, Ciappio ED, et al. Diet-induced obesity elevates colonic TNF-alpha in mice and is accompanied by an activation of Wnt signaling: a mechanism for obesity-as-sociated colorectal cancer. J Nutr Biochem. 2012;23(10): 1207-1213.

13. Lam YY, Ha CW, Campbell CR, et al. Increased gut permeabilityand microbiota change associate with mesenteric fat inflammation and metabolic dysfunction in diet-in-duced obese mice. PLoS One. 2012;7(3): e34233-34243.

14. Misra A, Singhal N, Khurana L. Obesity, the metabolic syndrome, and type 2 diabetes in developing countries: role of dietary fats and oils. J Am Coll Nutr. 2010; 29(3 suppl):289s-301s.

15. Zeyda M, Stulnig TM. Obesity, inflammation, and insulin resistance-A mini-review. Gerontology 2009;55(4): 379-386.

16. Manabe I. Chronic inflammation links cardiovascular, metabolic, and renal diseases. Circ J. 2011;75(12): 2739-2748.

17. Okwan-Duodu D, Umpierrez G.E, Brawley OW, et al. Obesity-driven inflammation, and cancer risk: role of myeloid derived suppressor cells and alternately activated macrophages. Am J Cancer Res. 2013;3(1): 21-33.

18. Tuominen I, Al-Rabadi L, Stavrakis D, et al. Diet-induced obesity promotes colon tumor development in azoxymethane-treated mice. PloS one 2013; 8(4):e60939.

19. Sharaf LK, Sharma M, Chandel D, et al. Prophylactic intervention of probiotics (L.acidophilus, L.rhamnosus GG) and celecoxib modulate Bax-mediated apoptosis in 1,2-dimethylhydrazine-induced experimental colon carcinogenesis. BMC cancer 2018; 18(1):1111.

20. Chandel D, Sharma M, Chawla V, et al. Isolation, characterization and identification of antigenotoxic and anticancerous indigenous probiotics and their prophylactic potential in experimental colon carcinogenesis. Scientific Reports. 2019; 9(1):14769.

21. Yang Y, Zhong Z, Wang B, et al. Early-life high-fat diet-induced obesity programs hippocampal development and cognitive functions via regulation of gut commensal Akkermansia muciniphila. Neuropsychopharmacology 2019;44(12):2054-2064

22. Zheng J, Zhang J, Guo Y, et al. Improvement on metabolic syndrome in high fat diet-induced obese mice through modulation of gut microbiota by sangguayin decoction. J Ethnopharmacol. 2020;246:112225

23. Tytgat HLP, Nobrega FL, van der Oost J, et al. Bowel biofilms: tipping points between a healthy and compromised gut? Trends in microbiology 2019; 27:17-25.

24. Kong F, Deng F, Li Y, et al. Identification of gut microbiome signatures associated with longevity provides a promising modulation target for healthy aging. Gut microbes 2019; 10:210-215 